\title{
Correlation between Age, Nodule Diameter, and TI-RADS Score in Thyroid Nodule Classification on Follicular Thyroid Carcinoma
}

\author{
Kania Difa Parama Citta ${ }^{1}$, Sahudi Sahudi ${ }^{2}$, Iskandar $\mathrm{Ali}^{2}$, \\ Marjono Dwi Wibowo ${ }^{2}$, Dwi Hari Susilo \\ ${ }^{1}$ General Surgery Resident, Surgery Faculty, Faculty of Medicine, Universitas Airlangga-Dr. Soetomo Teaching \\ Hospital, Surabaya, Indonesia \\ ${ }^{2}$ General Surgery Staff, Surgery Faculty, Faculty of Medicine, Universitas Airlangga-Dr. Soetomo Teaching \\ Hospital, Surabaya, Indonesia \\ Corresponding Author: Kania Difa Parama Citta
}

\begin{abstract}
Background: Thyroid cancer is a malignancy of the endocrine gland with the highest incidence. There are many radiological examination modalities that are used to help diagnose thyroid carcinoma, one of which is Ultrasonography. Ultrasonography (USG) can be useful to support the diagnosis of thyroid malignancy. A classification method that categorizes thyroid nodules based on risk for cancer, one of which is by using the Thyroid Imaging Reporting and Data System (TI-RADS). TI-RADS (Thyroid Imaging, Reporting and Data System) is a classification of thyroid ultrasound readings to differentiate between benign and malignant thyroid nodules. Several research efforts that have been done at Dr. Soetomo Hospital previously related to diagnostic of thyroid carcinoma but the results are meaningless and require large funds for the laboratory examination. The aim of this study is to make a relatively easy and inexpensive method using the TI-RADS classification, which is expected to assist in the preoperative diagnostics of a follicular thyroid carcinoma. It is hoped that there will be a method or modality that is easier, cheaper, accurate, and minimally invasive in predicting a follicular thyroid carcinoma.

Methods: In this cross-sectional study, we included patients with thyroid mass who underwent treatment in Surgery Department, Dr. Soetomo Teaching Hospital between January 2012 and December 2020. In this study, we utilized the patients' medical record to collect
\end{abstract}

the necessary clinical data. The inclusion criteria in this study were patients with singular thyroid nodule, underwent thyroid ultrasound, and diagnosed as follicular nodular carcinoma by histopathology examination. Finally, a total of 53 patients were included for further analysis. Ethical approval was obtained from the Ethics Committee of Dr. Soetomo Teaching Hospital (Surabaya, Indonesia).

Results: From a total of 53 research subjects, the subjects with the most age were more than 50 years old with a percentage of $52.8 \%$ or 28 patients and the rest, $47.2 \%$ or 25 patients. The results of this study indicates that nodule diameters less than $5 \mathrm{~cm}$ and more than $5 \mathrm{~cm}$ have almost the same number based on the number of data samples in this study, namely 53 patients. This can be seen from the number of respectively $27(50.9 \%)$ and $26(49 \%)$. In the TIRADS nodule score, the largest percentage obtained from medical data records in the form of a TIRADS score, namely a TIRADS score greater than TR 4 with a percentage of $60.4 \%$ or as many as 32 patients and the rest, namely a TIRADS score less than TR 4 of $39.6 \%$ or as much as 21 patients. In the third dependent variable, the authors looked for the odd ratio value for each variable on follicular carcinoma. The authors calculated the OR values for each variable, obtaining results of 1.012 for age, 1.111 for nodule size, and 3.520 for TIRADS scores.

Conclusion: There is a correlation between the TIRADS scores with the incidence of follicular thyroid carcinoma. 
Kania Difa Parama Citta et.al. Correlation between age, nodule diameter, and TI-RADS score in thyroid nodule classification on follicular thyroid carcinoma.

Keywords: Thyroid cancer, TIRADS, Follicular Thyroid Carcinoma

\section{INTRODUCTION}

Thyroid cancer is a malignancy of the endocrine gland with the highest incidence ${ }^{1}$. The incidence of thyroid cancer has increased rapidly in the United States (US) and other developed countries in the last 30 years. Follicular thyroid carcinoma accounts for $10 \%$ of thyroid cancers and is more common in iodine-deficient areas. Women had a higher incidence of follicular cancer, with a 3:1 female-to-male ratio, and a median age at presentation of 50 years. Based on histopathological examination, in Indonesia the incidence of papillary thyroid carcinoma is the highest $(33.1 \%)$, followed by follicular thyroid carcinoma (29.7\%), adenomatous goiter (22.3\%), and undifferentiated thyroid carcinoma (14.9\%) 2

There are many radiological examination modalities that are used to help diagnose thyroid carcinoma, one of which is Ultrasonography. Ultrasonography (USG) can be useful to support the diagnosis of thyroid malignancy. A classification method that categorizes thyroid nodules based on risk for cancer, one of which is by using the Thyroid Imaging Reporting and Data System (TI-RADS). TI-RADS (Thyroid Imaging, Reporting and Data System) is a classification of thyroid ultrasound readings to differentiate between benign and malignant thyroid nodules $3,4,5$.

Several research efforts that have been done at Dr. Soetomo Hospital previously related to diagnostic of thyroid carcinoma but the results are meaningless and require large funds for the laboratory examination. The aim of this study is to make a relatively easy and inexpensive method using the TI-RADS classification, which is expected to assist in the preoperative diagnostics of a follicular thyroid carcinoma. It is hoped that there will be a method or modality that is easier, cheaper, accurate, and minimally invasive in predicting a follicular thyroid carcinoma.

\section{METHODS}

\section{Study participants}

In this cross-sectional study, we included patients with thyroid mass who underwent treatment in Surgery Department, Dr. Soetomo Teaching Hospital between January 2012 and December 2020. In this study, we utilized the patients' medical record to collect the necessary clinical data. The inclusion criteria in this study were patients with singular thyroid nodule, underwent thyroid ultrasound, and diagnosed as follicular nodular carcinoma by histopathology examination. Finally, a total of 53 patients were included for further analysis. Ethical approval was obtained from the Ethics Committee of Dr. Soetomo Teaching Hospital (Surabaya, Indonesia).

Thyroid ultrasound and TIRADS Score, Thyroid ultrasound was performed on each patient prior to the surgery by experienced radiologist. Ultrasound was performed to evaluate the presence and characterize the thyroid nodule, such as the size, mass' internal structure, and vascularization. The American College of Radiology Thyroid Imaging Reporting and Data Systems (TIRADS) classification was used to evaluate the risk of cancer in thyroid nodules based on the characterization using ultrasound. TIRADS score was a 5-point scale ranged from 1-5; TIRADS 1 represented a normal thyroid gland, TIRADS 2 corresponded to a cystic benign or spongiform nodule. TIRADS 3 represented a benign nodule with no ultrasound suspicion. TIRADS 4 and above were corresponded to malignancy suspicion; TIRADS 4A was a low suspicion for thyroid malignancy and corresponded to several found: hypoechoic nodule with oval shape and no microcalcifications. TIRADS 4B was a high risk of malignancy, with shows feature of high malignancy suspicion: marked hypo-echogenicity, irregular margins, taller than wide shape, microcalcifications, low elasticity. TIRADS 5 nodules a strong predictor for thyroid malignancy and harboured three or more 
Kania Difa Parama Citta et.al. Correlation between age, nodule diameter, and TI-RADS score in thyroid nodule classification on follicular thyroid carcinoma.

features of high suspicion and/or a lymph node suspect of metastasis of thyroid origin.

\section{RESULTS}

From a total of 53 research subjects, the subjects with the most age were more than 50 years old with a percentage of $52.8 \%$ or 28 patients and the rest, $47.2 \%$ or 25 patients. The results of this study indicates that nodule diameters less than 5 $\mathrm{cm}$ and more than $5 \mathrm{~cm}$ have almost the same number based on the number of data samples in this study, namely 53 patients. This can be seen from the number of respectively $27(50.9 \%)$ and $26(49 \%)$. In the TIRADS nodule score, the largest percentage obtained from medical data records in the form of a TIRADS score, namely a TIRADS score greater than TR 4 with a percentage of $60.4 \%$ or as many as 32 patients and the rest, namely a TIRADS score less than TR 4 of $39.6 \%$ or as much as 21 patients. In the third dependent variable, the authors looked for the odd ratio value for each variable on follicular carcinoma. The authors calculated the OR values for each variable, obtaining results of 1.012 for age, 1.111 for nodule size, and 3.520 for TIRADS scores.

\begin{tabular}{|c|c|c|c|c|c|c|}
\hline \multirow{2}{*}{\multicolumn{2}{|c|}{ Variable }} & \multicolumn{2}{|c|}{20} & \multirow{4}{*}{$\begin{array}{l}\text { Mean } \\
47.37 \pm 12.871\end{array}$} & \multirow{4}{*}{$\begin{array}{l}\text { P value } \\
0,68\end{array}$} & \multirow{4}{*}{$\begin{array}{l}\text { OR 95\% CI } \\
1,012\end{array}$} \\
\hline & & \multirow{2}{*}{$\begin{array}{l}\mathbf{n}(\boldsymbol{\%}) \\
28(52,8 \%)\end{array}$} & \multirow{2}{*}{$\begin{array}{l}\text { Presentase } \\
52,8 \%\end{array}$} & & & \\
\hline \multirow[t]{2}{*}{ Usia } & $>50$ tahun & & & & & \\
\hline & $<50$ tahun & 25 & $47,2 \%$ & & & \\
\hline \multirow[t]{2}{*}{ Ukuran Nodul } & $>5 \mathrm{~cm}$ & 26 & $49 \%$ & \multirow[t]{2}{*}{$4.92 \pm 3.22$} & \multirow[t]{2}{*}{0,891} & \multirow[t]{2}{*}{1,111} \\
\hline & $<5 \mathrm{~cm}$ & 27 & $50,9 \%$ & & & \\
\hline \multirow[t]{2}{*}{ TIRADS } & $\geq 4$ & 32 & $60,4 \%$ & & \multirow[t]{2}{*}{0,039} & \multirow[t]{2}{*}{3,520} \\
\hline & 4 & 21 & $39,6 \%$ & & & \\
\hline
\end{tabular}

TIRADS thyroid nodules obtained the largest percentage from tracking medical record data in the form of TIRADS scores, namely TIRADS scores greater than TR 4 with a percentage of $60.4 \%$ or as many as 32 patients and the remaining TIRADS scores less than TR 4 by $39.6 \%$ or as many as 21 patients.

The results of this study indicate that nodule diameters less than $5 \mathrm{~cm}$ and more than $5 \mathrm{~cm}$ have almost the same number based on the number of data samples in this study, namely 53 patients. This can be seen from the number of respectively 27 (50.9\%) and $26(49 \%)$.

TIRADS thyroid nodules obtained the largest percentage from tracking medical record data in the form of TIRADS scores, namely TIRADS scores greater than TR 4 with a percentage of $60.4 \%$ or as many as 32 patients and the remaining TIRADS scores less than TR 4 by $39.6 \%$ or as many as 21 patients.

Based on the results of the Chi square test, it showed that the significance value was $0.680(\mathrm{P}>0.05)$, so there was no difference in age $<50$ and $>50$ as a diagnostic tool for follicular thyroid carcinoma. Based on the results of the Chi square test, the significance value was 0.891 $(\mathrm{P}>0.05)$, so there was no difference in tumor size $<5 \mathrm{~cm}$ and $5 \mathrm{~cm}$ as a diagnostic tool for follicular thyroid carcinoma. Based on the results of the Chi square test, it shows that the significance value is 0.039 ( $\mathrm{P}<$ $0.05)$, then there is a difference in the TIRADS score of TR $<4$ and TR 4 as a diagnostic tool for follicular thyroid carcinoma, the higher the TIRADS value with TR 4, the more determining thyroid carcinoma follicular.

The age data shows an abnormal distribution of data while the nodule size distribution data is normal, so it is continued with the Spearman test because there is an abnormal data distribution. Spearman's non-parametric correlation test was performed between age, nodule size and TIRADS of thyroid nodules against follicular thyroid carcinoma. In the nonparametric correlation test the result is no correlation because sig $>0.05$. 
Kania Difa Parama Citta et.al. Correlation between age, nodule diameter, and TI-RADS score in thyroid nodule classification on follicular thyroid carcinoma.

Tabel 5.2 - Tabel uji kolerasi non parametrik usia, ukuran nodul dan tirads nodul tiroid
\begin{tabular}{|l|l|l|l|l|l|}
\hline Correlations & Correlation Coefficient & 1.000 & .157 & -.024 \\
\hline \multirow{3}{*}{ Spearman } & \multirow{2}{*}{ Age } & Size & TIRADS \\
\cline { 3 - 6 } & & Sig &. & .261 & .864 \\
\cline { 3 - 6 } & $\mathrm{N}$ & 53 & 53 & 53 \\
\cline { 2 - 6 } & Size & Correlation Coefficient & .157 & 1.000 & -.076 \\
\cline { 3 - 6 } & Sig. & .261 &. & 0.591 \\
\cline { 3 - 6 } & $\mathrm{N}$ & 53 & 53 & 53 \\
\cline { 3 - 6 } & \multirow{3}{*}{ TIRADS } & Correlation Coefficient & -.024 & -.076 & 1.000 \\
\cline { 3 - 6 } & Sig & .864 & .591 &. \\
\cline { 3 - 6 } & $\mathrm{N}$ & 53 & 53 & 53 \\
\hline
\end{tabular}

\section{DISCUSSION}

The case of cancer in the thyroid gland is a malignancy of the endocrine gland with the highest incidence. The incidence of thyroid cancer has increased rapidly in the United States (US) and other developed countries in the last 30 years. Data from Surveillance, Epidemiology, and Final Results (SIER) found that from 1975 to 2009 there was a threefold increase in incidence, from 4.9 to 14.3 per 100,000 persons, while mortality was relatively constant at $\sim 0.5$ deaths per 100,000 people ${ }^{1}$. Meanwhile, in Indonesia the incidence is more in men than women with a percentage of $76.8 \%$ vs $23.2 \%{ }^{2}$.

Follicular carcinoma is the second most common cause of thyroid malignancy, with an incidence of around $15 \%$. The level of aggressiveness is greater than that of papillary thyroid malignancies. It occurs more often in women than men, occurs at an older age, especially in the 5-6 decade, and occurs mostly in endemic areas where iodine deficiency occurs and is rarely associated with a previous history of radiation to the neck area ${ }^{6,7}$.

Based on this study, it was found that from a total of 53 research subjects, the subjects with the most age were more than 50 years with a percentage of $52.8 \%$ or 28 patients and the rest were patients with age less than 50 years of $47.2 \%$ or as many as 25 patients. The results of this study are consistent with the data that the younger age group has less prevalence of malignancy than the elderly. Follicular thyroid carcinoma has a more frequent prevalence at an older age, especially in the 5-6 decade ${ }^{6,8}$.

The results of this study indicate that the examination using ultrasound modality to measure the diameter of nodules less than $5 \mathrm{~cm}$ and more than $5 \mathrm{~cm}$ has almost the same number based on the number of data samples in this study, namely 53 patients. This is different from the data from Lai et al. who said that the results of measurements of thyroid carcinoma measured using ultrasound equipment tended to or supported the diagnosis of carcinoma if the lesion size was larger ${ }^{9}$.

In the TIRADS nodule scoring, the largest percentage obtained from tracking medical record data in the form of a TIRADS score, namely a TR score greater than 4 with a percentage of $60.4 \%$ or as many as 32 patients and the rest, a TIRADS score of less than 4 by $39.6 \%$ or as many as 21 patients. These results are in accordance with the interpretation of the TIRADS score that ultrasound examination of thyroid nodules with reference to the TIRADS score system, TR 4 and TR 5 scores has a higher risk level for thyroid cancer. Based on the TIRADS interpretation, a score of TR 4 has a fairly suspicious meaning with an aggregate risk level of 5.9-12.8\%, while TR 5 has a very suspicious meaning with an aggregate risk level of $20.8 \%-68.4 \%$. TR 4 are patients with points 4-6 on the TIRADS scoring system while TR 5 are patients with points 7 or more. In this study, ACRTIRADS was used, which is a highly representative diagnostic aid, because it is based on the characteristics of the nodules, especially when performed by experienced radiologists who understand their use.

The author also looks at the distribution of data on two variables in this study, namely the variable size of the nodule and the variable TIRADS score. In the second presentation of the data, the author 
wants to see which variables are more representative of the occurrence of a malignancy. From the research data owned with nodule size $<5 \mathrm{~cm}$ there were 27 subjects $(50.9 \%)$ and nodule size $>5 \mathrm{~cm}$ there were 26 subjects (49\%). From a total of 27 subjects with a nodule size $<5 \mathrm{~cm}, 10$ subjects obtained TIRADS scores $<4$ and a TIRADS score 4 as many as 17 subjects. Based on these data, nodule size $>5 \mathrm{~cm}$ was found to have more TIRADS scores 4 as many as 15 subjects and 11 subjects for TIRADS scores <4. Based on the research data, the authors found that the TIRADS score data was more representative of the incidence of malignancy than the size of the nodule. This can be seen from the author's data on nodule size $<5 \mathrm{~cm}$ having TIRADS score data 4 . This data is supported by the theory that TIRADS scoring has several other assessment variables such as mass composition, echogenicity, shape size, margin limit, and calcification so that it is considered more comprehensive assessment of a nodule. In addition, TIRADS globally has a high sensitivity to detect malignancies ranging from $92.4 \%$. So, the authors believe that TIRADS examination is considered better than just looking at the size of the nodule in cases of thyroid malignancy ${ }^{4,5}$.

In addition to the size of the thyroid nodule, in this study the patient was also subjected to ultrasound examination to assess the TIRADS score. There were 17 patients $(32 \%)$ with thyroid nodule size $<5$ $\mathrm{cm}$ but had TIRADS score 4 and histopathological results were follicular thyroid carcinoma. In principle, TIRADS is a diagnostic tool and as a predictor of a nodule that leads to malignancy or benign. However, as a diagnostic tool, it certainly has weaknesses because it only looks at the characteristics of the nodule structure via the ultrasound probe and has a subjective operator dependent value. In a study by Schenke et al 2020, it was found that even if you have a TIRADS score $<4$ there is still the possibility of a malignancy occurring, but the lower the patient's TIRADS score, the less likely it will occur. It can be concluded that a TIRADS score $<4$ does not guarantee a nodule does not lead to malignancy, but a lower probability than a TIRADS score $>4^{10}$.

From this study, there were 12 patients $(22.6 \%)$ with thyroid nodule size < $5 \mathrm{~cm}$ with histopathological results of thyroid follicular carcinoma but benign FNAB. The same thing was found in a study conducted by Karadeniz, et al in 2019, in that study it was stated that the size of thyroid nodules was not associated with a tendency to follicular thyroid carcinoma. In this study, it was found that nodule size $>5$ $\mathrm{cm}$ had more false negative results based on FNAB examination, which was more than $50 \%(70 \%)$. (Karadeniz et al., 2019) When compared to this study, there were 17 patients $(32 \%)$ with nodule size $>5 \mathrm{~cm}$ with FNAB results not suggesting malignancy but histopathological results were follicular thyroid carcinoma (false negative). In detail, there were 16 patients $(30.1 \%)$ with nodule size $>5 \mathrm{~cm}$ who had histopathological results of an encapsulated follicular variants of papillary thyroid. So that the size of the thyroid nodule cannot be used as the only parameter as a predictor of follicular thyroid carcinoma.

Based on the results of this study, the authors obtained data that among the three variables carried out by the study, the first variable was age which was grouped into two, namely under 50 years and above 50 years. Then the second variable was the size of the thyroid nodules which the authors divided into two groups, namely the size of the thyroid nodules. $<5 \mathrm{~cm}$ and thyroid nodule size $>5 \mathrm{~cm}$, and the last variable is the TIRADS score divided into two groups, namely the TR value $<4$ and the TR value 4 . In this study the authors wanted to see the relationship between the three independent variables and the dependent variable, namely the incidence of follicular carcinoma. . Based on the results of the study, it was found that of all the independent variables studied by the authors, the TIRADS score with a TR value of 4 had a significant meaning and 
relationship with follicular carcinoma while the thyroid nodule size and age variables did not find a significant relationship. Therefore, TIRADS score 4 is not associated with thyroid nodule size and patient age with follicular thyroid carcinoma. The results of the research conducted by the authors are in line with research conducted by Hong, Min Ji 2018 that similar data were obtained, namely there was no mutually reinforcing relationship between TIRADS scores, nodule size, and patient age for follicular thyroid carcinoma. The study conducted by Hong, Min Ji was also similar in that only the TIRADS score variable had a positive meaning or relationship with the incidence of follicular thyroid carcinoma. The higher the TIRADS score, the greater the probability that a person will develop follicular thyroid carcinoma.

The effect of age on the incidence of follicular thyroid carcinoma is still a matter of debate. The follicular type of thyroid carcinoma is more common in older patients than the papillary type. Follicular thyroid carcinoma appears mostly in women and the elderly with a female: male ratio of 3:1 and the mean age is 60 years ${ }^{11,12,13}$.

The author considers that a study with larger data is needed in order to represent age risk factors for the incidence of follicular thyroid carcinoma. Next, the writer performs calculations to find the OR value for each variable. There were significant results on the TIRADS score with follicular carcinoma with an OR value of 3,520. This means that there is a 3.5 -fold possibility in patients with thyroid nodules and having a TIRADS score 4 for follicular carcinoma. Meanwhile, for age and nodule size, the OR was 1.012 for age and 1.111 for nodule size.

In diagnosing a follicular thyroid carcinoma, it cannot only be judged by the size of the thyroid nodule and FNAB alone. Patients with thyroid nodules should be considered for clinical assessment, ultrasound examination results, and TIRADS scores. So that with an accurate and good preoperative diagnosis the patient can avoid the risk of inadequate treatment. An example is more than one operation on the thyroid; this can cause fibrotic tissue in the thyroid which makes it difficult for the surgeon to identify the $\mathrm{n}$. recurrent laryngeal and parathyroid glands. So that repeated operations have two of the most common risks, namely injury to $n$. laryngeal recurrences and elevation of the parathyroid glands ${ }^{13,15}$.

\section{CONCLUSION}

There is a correlation between the TIRADS scores with the incidence of follicular thyroid carcinoma.

\section{Conflict of Interest}

No competing interests declared.

\section{Acknowledgement: None}

\section{Source of Funding: None}

\section{Ethical Approval: Approved}

\section{REFERENCES}

1. Nguyen, Q. T. Lee, E. J. Huang, M. G. Park, Y. I. Khullar, A. et al. (2015) 'Diagnosis and treatment of patients with thyroid cancer', American Health and Drug Benefits, 8(1), pp. 30-38.

2. Kartini \& Wibisana. 2017. Accuracy of Triple Diagnostic Test in Patients with Thyroid Nodule at Dr Cipto Mangunkusumo General Hospital. e JKI: Vol. 5 No. 1, April 2017

3. Chaigneau, E. Russ, G. Royer, B. Bigorgne, C. Bienvenu-Perrard, M. et al. (2018). TIRADS score is of limited clinical value for risk stratification of indeterminate cytological results. European Journal of E ndocrinology, 179(1), pp. 13-20

4. Borlea, A., Borcan, F., Sporea, I., Dehelean, C. A., Negrea, R., Cotoi, L., \& Stoian, D. (2020). TI-RADS Diagnostic Performance: Which Algorithm is Superior and How Elastography and 4D Vascularity Improve the Malignancy Risk Assessment. Diagnostics (Basel, Switzerland), 10(4), 180

5. Rahal, A. Falsarella, P. M. Rocha, R. D. Lima, J. P. B. C. Lani, M. J. et al. (2016) 
Kania Difa Parama Citta et.al. Correlation between age, nodule diameter, and TI-RADS score in thyroid nodule classification on follicular thyroid carcinoma.

'Correlation of Thyroid Imaging Reporting and Data System [TI-RADS] and fine needle aspiration: experience in 1,000 nodules', Einstein (Sao Paulo, Brazil), 14(2), pp. 119-123

6. Wijayahadi, Yoga; Panuwun, Tulus; dan Harwindo, Bramantyo. Ekspresi CDK4 sebagai predictor keganasan pada neoplasma tiroid tipe folikuler. 2013

7. Kakudo, K. Bychkov, A. Bai, Y. Li, Y. Liu, Z. Jung, C. (2018) 'The new 4th edition World Health Organization classification for thyroid tumors, Asian perspectives', Pathology International, 68(12), pp. 641-64

8. Haugen B, Alexander E, Bible K, Doherty G, Mandel S, Nikiforov Y et al. 2015. American Tiroid Association Management Guidelines for Adult Patients with Tiroid Nodules and Differentiated Tiroid Cancer: The American Tiroid Association Guidelines Task Force on Tiroid Nodules and Differentiated Tiroid Cancer. Tiroid. 2016;26(1) pp.1-133

9. Lai, X. Jiang, Y. Zhang, B. Liang, Z. Jiang, Y. et al. (2018) 'Preoperative sonographic features of follicular thyroid carcinoma predict biological behavior A retrospective study', Medicine (United States), 97(41), pp. 1-6

10. Schenke, S. Klett, R. Seifert, P. Kreissl, M. C. Gorges, R. Zimny, M. (2020) 'Diagnostic Performance of Different Thyroid Imaging Reporting and Data Systems (KwakTIRADS, EU-TIRADS and ACR TIRADS) for Risk Stratification of Small Thyroid Nodules $(10 \mathrm{~mm})$ ', Journal of Clinical Medicine, 9(1), p. 236.
11. American Cancer Society. Tiroid cancer: what are the key statistics about tiroid cancer Revised January 12, 2015. (Diakses pada www.cancer.org/cancer/tiroidcancer/detaile dguide/tiroid-cancer-key-statistics)

12. D'Avanzo A, Treseler P, Ituarte PHG, Wong M, Streja L, Greenspan FS, et al. Follicular thyroid carcinoma: histology and prognosis. Cancer. 2004 Mar;100(6):1123-9

13. Besic, N. Zgajnar, J. Hocevar, M. FrkovicGrazio, S. (2005). Is patient's age a prognostic factor for follicular tiroid carcinoma in the TNM classification system'. Tiroid, 15(5), pp. 439-48.

14. Brunicardi, F.C. Andersen, D.K. Biliar, T.R. Dunn, D.L. Hunter, J.G. et al. (2019) Schwartz Principles of Surgery,11th ed. USA: McGraw-Hill

15. Sy, A. et al. (2017) 'Indications and Morbidity of Reoperative Thyroid Surgeries in a Military Hospital of Senegal', International Journal of Otolaryngology, 2017, pp. 1-5. doi: 10.1155/2017/4045617

16. Bates, M. F. Lamas, M. Randle, R. Long, K. Pitt, S. et al. (2018). Back so soon? Is early recurrence of papillary tiroid cancer really just persistent disease. Surgery (United States), 163(1), pp. 118-23.

How to cite this article: Citta KDP, Sahudi S, Iskandar Ali et.al. Correlation between age, nodule diameter, and TI-RADS score in thyroid nodule classification on follicular thyroid carcinoma. International Journal of Research and Review. 2021; 8(8): 385-391. DOI: https://doi.org/10.52403/ijrr.20210853 